\title{
Tracking the proteome in specific cell-types in vivo
}

Erin Schuman is interested in the proteome, and for over ten years she and her colleagues have been working to develop alternative methods for labeling and identifying newly synthetized proteins in specific cell types. In a new report in Nature Biotechnology from her group at Max Plank Institute for Brain Research and colleagues, they present a new mouse line that, when crossed with Cre driver lines, can label newly created proteins with cell-type specificity that could prove invaluable to the study of degenerative disease models (Nat. Biotechnol. doi:10.1038/nbt.4016; published online 06 November 2017).

When working at Caltech with David Tirrell and Daniela Dieterich several years back, Schuman helped develop a cell-type specific metabolic labeling method with the methionine surrogate azidonorleucine through expression of a mutant methionyl-tRNA synthetase, which they termed MetRS. Now, building on this method, they have moved it in vivo, developing a mouse line that can be crossed with any Cre-driver lines to for labeling nascent proteins. The group tested and validated their mouse line by crossing with lines to label proteins in excitatory principle neurons and Purkinje neurons. Using an environmental enrichment strategy, they also discovered over 200 proteins that were differentially regulated within the hippocampus (excitatory neurons), demonstrating the power to link behavioral changes directly with cell-type specific proteomic changes.

"The challenge wasn't so much in the construction of the mouse as in the optimizing the mass spectroscopy aspect of it," says Schuman. "The chemistry that is used in the in vivo setting is much more challenging than doing things in vitro, so we spent a lot of time optimizing that methodology to get the high protein number and the low background numbers that we knew we wanted and that makes the whole thing worthwhile."
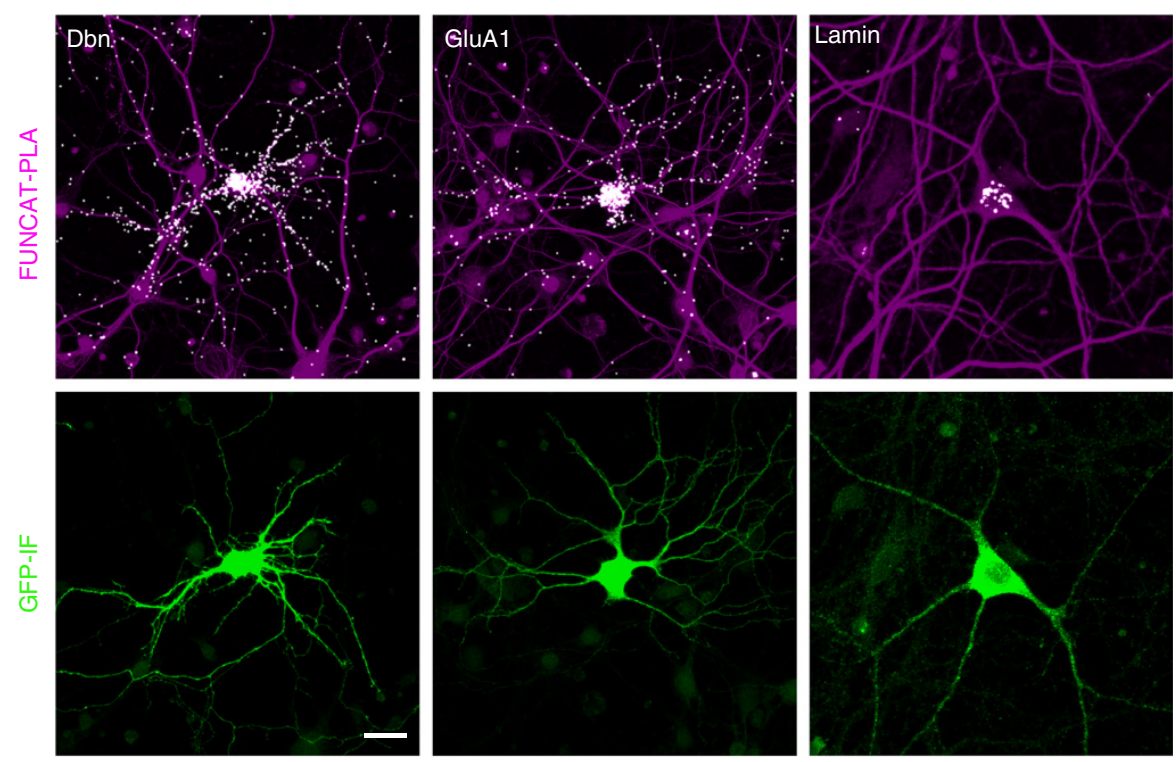

Subcellular localization of newly synthesized Drebrin (Dbn), GluA1, and Lamin proteins in cultured hippocampal neurons using new method, described in Nat. Biotechnol. doi:10.1038/nbt.4016; 2017.

To identify proteins in the brain in vivo, after MetRS labeling of newly synthesized proteins in the cells of interest (driven by Cre), brain tissue was then taken and processed using one of two steps, FUNCAT (fluorescent non-canonical amino-acid tagging) for imaging, or BONCAT (bio-orthogonalnon-canonical amino-acid tagging) for quantifying using mass spec. Using gene ontology analysis, the research team was able to identify several disease-related proteins in the hippocampus, demonstrating the technique's potential to monitor, in vivo, clinically important components of the proteome.

Schuman has high hopes for the new mouse line, in her lab and beyond. "We've already got a bunch of collaborations set up, and we've deposited the mouse at Jackson Laboratories, so I think there is going to be a huge potential for this in all kinds of disease-model settings," she says. In her sights are potential developments for understanding disease progression in Parkinson's disease models. "One thing we are trying to look at now is the dopaminergic proteome. Dopaminergic cells are important for lots of diseases, notably Parkinson's disease, so we'll be able to see what the dopaminergic cells in the substantia nigra look like and then by crossing to one or even all of the Parkinson's mouse models we could actually see what the disease proteome looks like in a particular cell class."

Additionally, she notes how the new mouse line and methods her team has developed enable researchers to track the temporal changes in the proteome. "You could see how things change over time, which is important because a lot of these neurodegenerative diseases don't manifest in the first month or so symptomatically. But we don't know when the proteomic changes occurs, so you could get not only a view of the disease, but how it evolves over time in a particular cell type. I think that's going to be really important and critical for the whole field."

Dustin M. Graham 